\title{
Relativistic Effects in Exclusive pd Breakup Scattering at Intermediate Energies
}

\author{
T. Lin ${ }^{\mathrm{a}}$, Ch. Elster ${ }^{\mathrm{a}, \mathrm{b}}$, W. N. Polyzou ${ }^{\mathrm{c}}$, W. Glöckle ${ }^{\mathrm{d}}$, \\ ${ }^{a}$ Institute of Nuclear and Particle Physics, and Department of Physics and Astronomy, Ohio \\ University, Athens, OH 45701, USA \\ b Physics Division, Argonne National Laboratory, Argonne, IL 60439, USA \\ ${ }^{\mathrm{c}}$ Department of Physics and Astronomy, The University of Iowa, Iowa City, IA 52242, USA \\ dinstitute for Theoretical Physics II, Ruhr-University Bochum, D-44780 Bochum, Germany
}

\begin{abstract}
The relativistic Faddeev equation for three-nucleon scattering is formulated in momentum space and directly solved in terms of momentum vectors without employing a partial wave decomposition. Relativistic invariance is achieved by constructing a dynamical unitary representation of the Poincaré group on the three-nucleon Hilbert space. The exclusive breakup reaction at $508 \mathrm{MeV}$ is calculated based on a Malfliet-Tjon type of two-body interaction and the cross sections are compared to measured cross sections at this energy. We find that the magnitude of the relativistic effects can be quite large and depends on the configurations considered. In spite of the simple nature of the model interaction, the experimental cross sections are in surprisingly good agreement with the predictions of the relativistic calculations. We also find that although for specific configurations the multiple scattering series converges rapidly, this is in general not the case.
\end{abstract}

Key words: Relativistic Quantum Mechanics, Faddeev Equation, The Quantum Mechanical Three-Body Problem, n-d Scattering

PACS: 21.45+v, 24.10.Jv, 25-10.+s

Breakup reactions in the proton-deuteron $(p d)$ system at intermediate energies have been studied experimentally quite intensively in recent decades. A prominent set of data can be found in the comprehensive overview of the experiments completed at Saturne2 [1]. However, the theoretical interpretation faced and still faces serious challenges. At those energies pion production channels are open and nuclear resonances play a role. In contrast to the energy regime below the pion threshold, where high precision nucleonnucleon $(\mathrm{NN})$ forces are established [2]4, and nuclear forces based on effective chiral dynamics are being developed [5], the intermediate energy regime, in which the lowest 
nucleon resonances play a role, does not yet have forces describing data with comparable quality [6]. Moreover it can be expected that three-nucleon (3N) forces will play a more prominent role in the intermediate energy regime. The tendency for an increasing importance of $3 \mathrm{~N}$ forces with increasing energy has already been seen in $3 \mathrm{~N}$ observables at low energies [7.

A consistent treatment of intermediate energy reactions requires a Poincaré symmetric quantum theory [8]. In addition, the standard partial wave decomposition, successfully applied below the pion-production threshold [9], is no longer an adequate numerical scheme due to the proliferation of the number of partial waves. Thus, the intermediate energy regime is a new territory for few-body calculations, which waits to be explored.

The aim of this article is to address two aspects in that list of challenges: exact Poincaré invariance and calculations using vector variables instead of partial waves. In a series of publications [10 the technique of solving the nonrelativistic momentum-space Faddeev equation without partial waves has been mastered, for bound as well as scattering states. The Faddeev equation, based on a Poincaré invariant mass operator, has been formulated in detail in [11. The resulting Faddeev equation has both kinematical and dynamical differences with respect to the corresponding nonrelativistic equation.

The formulation of the theory is given in a representation of Poincaré invariant quantum mechanics where the interactions are invariant with respect to kinematic translations and rotations [12. The model Hilbert space is a three-nucleon Hilbert space (thus not allowing for absorptive processes). The method used to introduce the NN interactions in the unitary representation of the Poincaré group allows to input high-precision NN interactions 234 in a way that reproduces the measured two-body observables. However in this study we use a simpler interaction consisting of a superposition of an attractive and a repulsive Yukawa interaction that supports a bound state with the deuteron binding energy. Poincaré invariance and $S$-matrix cluster properties dictate how the twobody interactions must be embedded in the three-body dynamical generators. Scattering observables are calculated using Faddeev equations formulated with the mass Casimir operator (rest Hamiltonian) constructed from these generators.

In [1] the driving term in the relativistic Faddeev equation (first order in the two-body transition operator) has been used to evaluate $p d$ elastic scattering as well as break-up cross sections. This has now been completed by fully solving the relativistic Faddeev equation based on the numerical techniques previously used to solve the nonrelativistic Faddeev equation 10. Our calculations converge well up to $2 \mathrm{GeV}$, indicating the applicability of the formulation of the Faddeev equation based on vector variables for intermediate energies. We want to point out that the relativistic Faddeev equations with realistic spin-dependent interactions have been solved below the pion-production threshold in 13 a partial wave basis.

In order to estimate the size of relativistic effects the interactions employed in the nonrelativistic and relativistic calculations presented here are chosen to be phase shift equivalent. This is achieved in this article by adding the interaction to the square of the mass operator [1516]. In this comparison differences in the relativistic and nonrelativistic calculations first appear in the three-body calculations. Those differences are in the choice of kinematic variables (Jacobi momenta are constructed using Lorentz boosts rather than Galilean boosts) and in the embedding of the two-body interactions in the threebody problem, which is a consequence of the non-linear relation between the two and three-body mass operators. These differences modify the permutation operators and the 
off-shell properties of the kernel of the Faddeev equations.

The relativistic Faddeev equation is solved in the form

$$
T(z)=t(z) P+t(z) P\left(z-M_{0}\right)^{-1} T(z),
$$

where $P$ is a permutation operator and $t(z)$ is the two-body transition operator embedded in the three-nucleon Hilbert space defined as the solution to

$$
t(z)=V+V\left(z-M_{0}\right)^{-1} t(z) .
$$

Here the interaction is given as $V=M-M_{0}$, where $M$ is the three-particle mass operator with one two-body interaction alone and $M_{0}$ the corresponding free mass operator. The physical transition amplitudes for elastic scattering and the breakup processes can be expressed in terms of $T(z)$. Our aim in this article is to calculate exclusive threebody breakup processes in the intermediate energy region and compare relativistic and nonrelativistic results. Since we generate the solution of the Faddeev equation from the multiple scattering series (resulting from iterating Eq. (10), we can also obtain insight into the contributions of the various orders of that series. The exact solution to the Faddeev equation is constructed from the multiple scattering series using Padé summation.

Though our two-body force is simple, we want to compare to a ${ }^{2} \mathrm{H}(\mathrm{p}, 2 \mathrm{p}) \mathrm{n}$ experiment at a projectile kinetic energy $508 \mathrm{MeV}$ [17] to see if our calculation captures essential features of the measurement. Differences in the predictions of our relativistic and nonrelativistic calculations are already very pronounced at this energy.

The five-fold laboratory differential cross section for exclusive scattering is given by 18 .

$$
\begin{aligned}
\frac{d^{5} \sigma_{b r}^{l a b}}{d \Omega_{1} d \Omega_{2} d E_{1}}= & (2 \pi)^{4} \frac{E\left(q_{0}\right) E_{d}\left(q_{0}\right) E(q)}{2 k_{l a b} m_{d}} \frac{p_{1} p_{2}^{2}}{p_{2}\left(\mathrm{E}-E\left(p_{1}\right)\right)-E\left(p_{2}\right)\left(\mathbf{P}-\mathbf{p}_{1}\right) \cdot \hat{\mathbf{p}}_{2}} \\
& \times E(k) \sqrt{4 E^{2}(k)+\mathbf{q}^{2}}\left|\left\langle\mathbf{k}, \mathbf{q}\left\|U_{0}\right\| \varphi_{d}, \mathbf{q}_{0}\right\rangle\right|^{2} .
\end{aligned}
$$

Here $U_{0}=(1+P) T$ is the operator for breakup scattering, $\mathrm{E}$ is the total energy of the system and $\mathbf{P}$ its total momentum.

The results of our calculations are displayed in Figs. 1, 4. In Fig. 1 we chose selected outgoing proton laboratory angle pairs $\theta_{1}-\theta_{2}$ from Ref. 17, which are symmetric around the beam axis. The cross sections are plotted against the laboratory kinetic energy of one of the outgoing protons. It is interesting to see that for the smaller angle pairs, $\theta_{1,2}=38.1^{\circ}$ and $41.5^{\circ}$, the relativistic cross sections (solid lines) are considerably larger than the nonrelativistic ones (dashed lines). For angle pairs around $\theta_{1,2}=44^{\circ}$ and larger this reverses and the relativistic cross sections fall below the nonrelativistic ones. A similar phenomenon has been observed in Ref. [14 for a projectile energy of $200 \mathrm{MeV}$, where the relativistic Faddeev equation has been solved based on the CD-Bonn NN force. Surprisingly, our present relativistic calculations come close to the data 17. The fact that the first order results nearly coincide with the full calculation may be explained by the quasi free scattering (QFS) condition, which however, is realized only for the angle pair $\theta_{1,2}=41.5^{\circ}-41.4^{\circ}$.

Configurations in which the outgoing protons are measured asymmetric with respect to the beam axis are shown in Fig. 2. Again, the relativistic effects are very pronounced in all configurations shown. The angle combination $30.1^{\circ}-53.7^{\circ}$ is the only QFS condition. 
In order to illustrate how the cross section is built up by the lowest order terms of the multiple scattering series, we show in Fig. 3 the relativistic results for the first order in the two-body t-matrix, then add successively one and two rescattering terms, and compare to the full calculation. For the QFS condition, $41.5^{\circ}-41.4^{\circ}$, rescattering terms do not play a role, whereas in the two asymmetric configurations even the 3rd order in the multiple scattering series is not quite sufficient to arrive at the full result over the entire measured energy range.

To explore the importance of higher order terms in the multiple scattering series, we display in Fig. 4 three configurations for the coplanar star and one for the space star. (In the star configurations the interparticle pair angles are $120^{\circ}$ in the center of momentum (c.m.) system). The angles $x_{q}=\hat{q} \cdot \hat{q}_{0}$ and $x_{p}=\hat{p} \cdot \hat{q}_{0}$ refer to the c.m. system with $\mathbf{p}$ and $\mathbf{q}$ being Poincare-Jacobi momenta and $\mathbf{q}_{\mathbf{0}}$ being the initial particle momentum. The angle $\phi_{p q}=0^{\circ}$ indicates coplanar star configurations, while $\phi_{p q}=90^{\circ}$ indicates a space star configuration. For $x_{q}=1$ (one particle being ejected forward along the direction of qo) the first order result is completely misleading and about two orders of magnitude higher than the fully converged result, which in turn requires more than four orders in the multiple scattering series. However, the first order clearly shows the shift in the peak position characteristic for relativistic calculations [11. The configuration $x_{q}=0.5$ also exhibits this shift in the peak position when comparing relativistic and nonrelativistic calculation, and again the full result is built up by several rescattering contributions. The coplanar star at $x_{q}=-1$ exhibits the QFS condition (in the laboratory frame particle 1 would stay at rest), and thus the first order is sufficient. In addition there is no shift in the peak position due to relativistic effects, which can be understood as consequence of the particle being at rest. In case of the space star, the first order does not contribute, and the cross section of this configuration is slowly built up by rescattering contributions, requiring orders higher than four. The star configuration shown is representative for all space star configurations achieved by rotation in the plane perpendicular to the beam.

These observations suggest that even at intermediate energies the full solution of the Faddeev equation is needed to make reliable predictions. There is no loss of generality in using this formalism: because the two-nucleon interactions are fit to two-nucleon data and $S$-matrix cluster properties are used to embed the two-nucleon interactions in the three-nucleon system, any other Poincaré invariant three-nucleon model with these properties differs from this one by at most a three-body interaction. The difference between the relativistic and nonrelativistic calculations using the same two-body input first appears in the three-body system. Our calculation indicates measurable differences in the corresponding relativistic and nonrelativistic breakup cross sections. Even though our model interaction is much simpler than a realistic two-body interaction, the exclusive breakup cross section are in surprisingly good agreement with existing data at $508 \mathrm{MeV}$. The results reported in this letter suggest that

(a) A direct integration without employing a partial wave decomposition can successfully be used to solve the relativistic Faddeev equations with embedded NN interactions.

(b) The formulation of Poincaré invariant quantum theory with a three-dimen-sional kinematic Euclidean symmetry is a suitable formalism for treating scattering in the intermediate energy region. This is supported by the surprising agreement with the measurements reported in [17.

(c) Convergence of the multiple scattering series is not guaranteed at intermediate energies. 
A detailed description of our results comprising elastic, inclusive and exclusive processes will follow in a subsequent publication.

Acknowledgments This workwas performed in part under the auspices of the $\mathrm{U}$ S. Dewith Ohio University, contract No. DE-FG02-86ER40286 with the University of Iowa, and contract No. DE-AC02-06CH11357 with Argonne National Laboratory. The authors would like to acknowledge fruitful discussions with D.R. Phillips contributing to this work, and thank V. Punjabi for providing the experimental data. C.E. acknowledges the hospitality of the Institute of Modern Physics (IMP) in Lanzhou, China, where this work was initiated. We thank the Ohio Supercomputer Center (OSC) for the use of their facilities under grant PHS206.

\section{References}

[1] The 20 years of the synchrotron Saturn-2, eds. A. Boudard, P.A. Chamouard, World Scientific, 2000.

[2] R.B. Wiringa, V.G.J. Stoks, R. Schiavilla, Phys. Rev. C51, 38 (1995).

[3] R. Machleidt, Phys. Rev. C63, 024001 (2001).

[4] V.G.J. Stoks, R.A.M. Klomp, C.P.F. Terheggen, and J.J.de Swart, Phys. Rev. C49, 2950 (1994).

[5] E. Epelbaum, Prog. Part. Nucl. Phys. 57654 (2006).

[6] A. Pricking, Ch. Elster, A. Gardestig and F. Hinterberger [EDDA Collaboration], arXiv:0708.3692 [nucl-th].

[7] H. Witala, W. Glöckle, J. Golak, A. Nogga, H. Kamada, R. Skibinski, and J. Kuros-Zolnierczuk, Phys. Rev. C63, 024007 (2001).

[8] E.P. Wigner, Ann. Math. C 40, 149 (1939).

[9] W. Glöckle, H. Witala, D. Hüber, H. Kamada, and J. Golak, Phys. Rep. 274, 107 (1996).

[10] Ch. Elster, J.H. Thomas, and W. Glöckle, Few-Body Systems 24, 55 (1998); Ch. Elster, W. Schadow, A. Nogga, and W. Glöckle, Few-Body Systems 27, 83 (1999); H. Liu, Ch. Elster, and W. Glöckle, FewBody Systems 33, 241 (2003); H. Liu, Ch. Elster, and W. Glöckle, Phys.Rev. C72, 054003 (2005).

[11] T. Lin, Ch. Elster, W. N. Polyzou and W. Glöckle, Phys. Rev. C 76, 014010 (2007).

[12] F. Coester, Helv. Phys. Acta. 38, 7 (1965).

[13] H. Witala, J. Golak, W. Glöckle, H. Kamada, Phys. Rev. C71, 054001 (2005); H. Witala, J. Golak, R. Skibinski, Phys. Lett. B 634, 374 (2006); H. Witala, R. Skibinski, J. Golak, Eur. Phys. J. A 29, 141 (2006).

[14] R. Skibinski, H. Witala and J. Golak, Eur. Phys. J. A 30, 369 (2006).

[15] F. Coester, S.C. Pieper, F.J.D. Serduke, Phys. Rev. C11, 1 (1975).

[16] B.D. Keister and W.N. Polyzou, Phys. Rev. C 73, 014005 (2006).

[17] V. Punjabi et al., Phys. Rev. C 38, 2728 (1988).

[18] Note that in 11] the five-fold differential breakup cross sections contain an erroneous factor of $1 / 3$. 


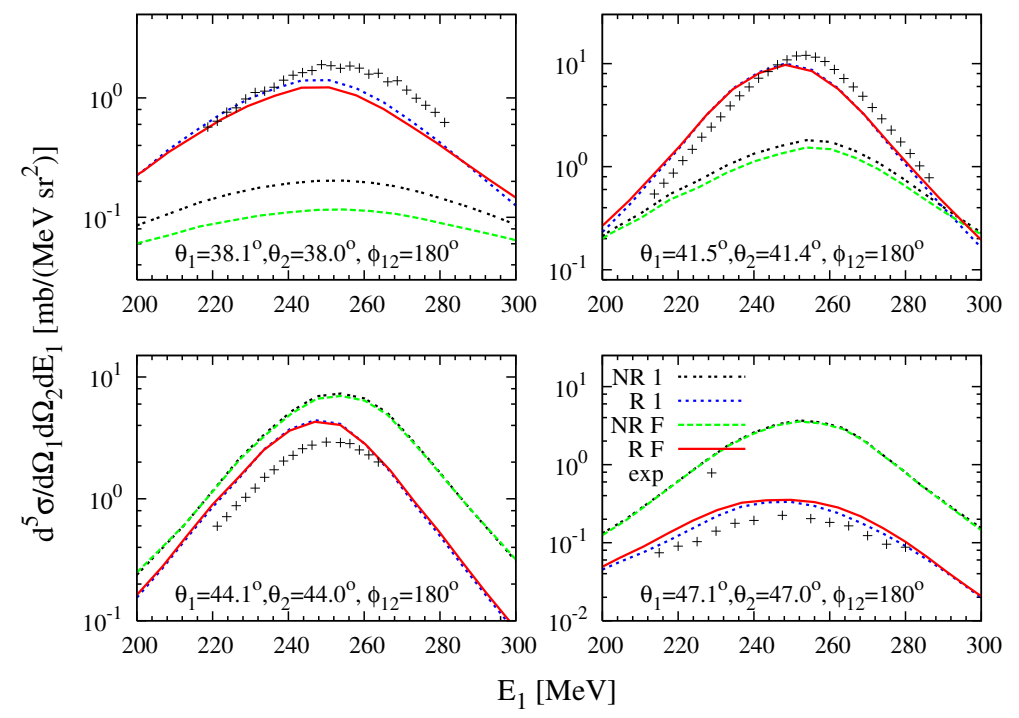

Fig. 1. The exclusive differential cross section for the ${ }^{2} \mathrm{H}(\mathrm{p}, 2 \mathrm{p}) \mathrm{n}$ reaction at $508 \mathrm{MeV}$ laboratory projectile energy for different proton angle pairs $\theta_{1}-\theta_{2}$ symmetric around the beam axis as function of the laboratory kinetic energy of one of the outgoing protons. The solid line ( $\mathrm{R} \mathrm{F}$ ) represents the full relativistic solution of the Faddeev equation, while the dotted curve (R 1) indicates the relativistic calculation based on the 1st order in the multiple scattering expansion of the Faddeev amplitude. The corresponding nonrelativistic full solution of the Faddeev equation is given by the short-dashed curve (NR F) and its 1st order contribution by the double-dotted curve (NR 1). The data are taken from Ref. [17]. 


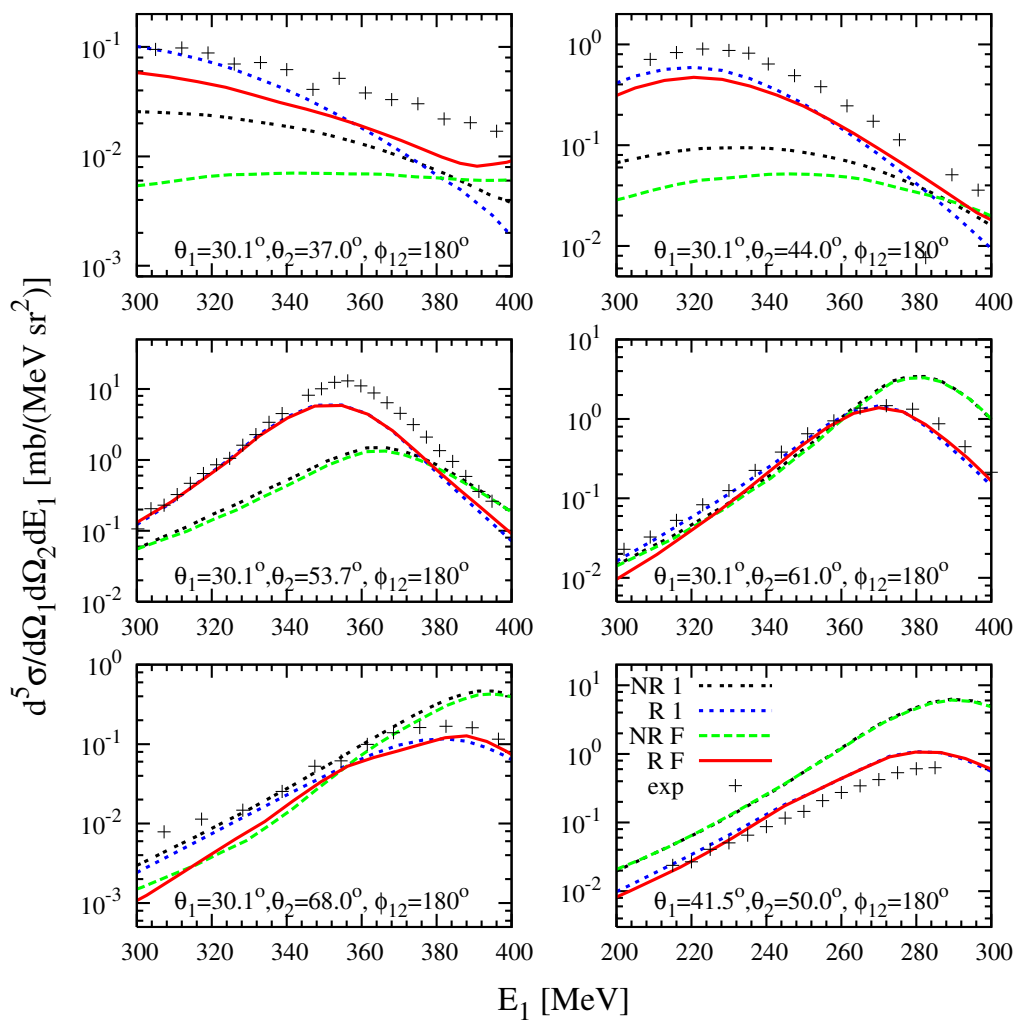

Fig. 2. The exclusive differential cross section for the ${ }^{2} \mathrm{H}(\mathrm{p}, 2 \mathrm{p}) \mathrm{n}$ reaction at $508 \mathrm{MeV}$ laboratory projectile energy for different proton angle pairs $\theta_{1}-\theta_{2}$ asymmetric around the beam axis as function of the laboratory kinetic energy of one of the outgoing protons. The meaning of the curves is the same as in Fig. [1] The data are taken from Ref. [17]. 


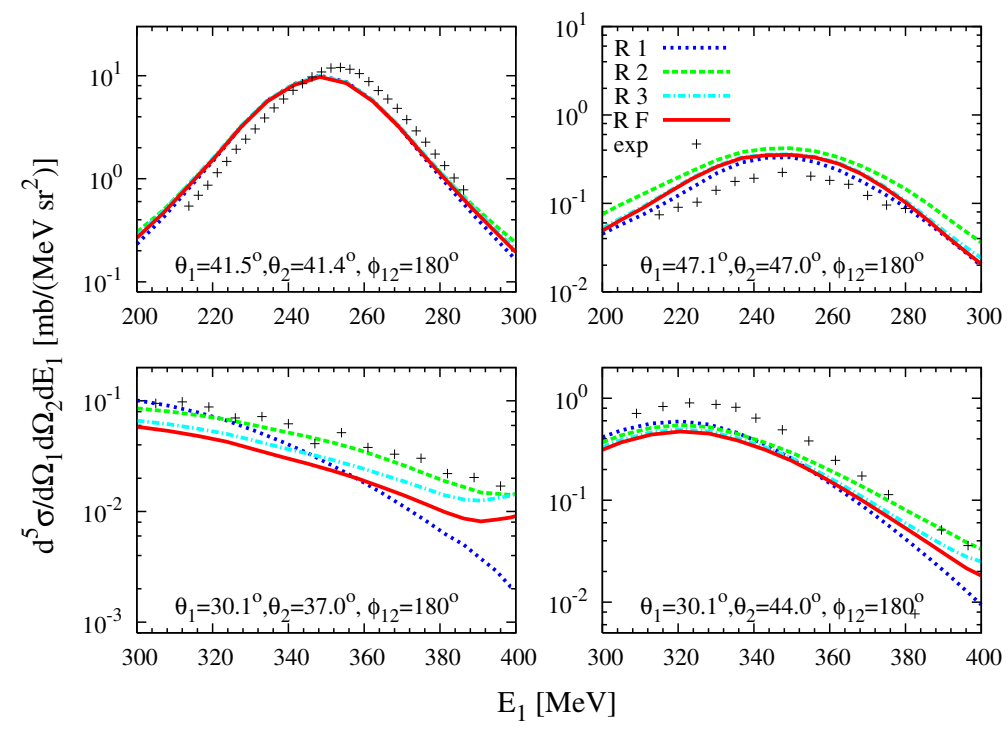

Fig. 3. The exclusive differential cross section for the ${ }^{2} \mathrm{H}(\mathrm{p}, 2 \mathrm{p}) \mathrm{n}$ reaction at $508 \mathrm{MeV}$ laboratory projectile energy for different proton angle pairs $\theta_{1}-\theta_{2}$ as function of the laboratory kinetic energy of one of the outgoing protons. The solid line (R F) represents the full relativistic solution of the Faddeev equation. The dotted line ( R 1) represents the relativistic calculation based on the 1st order term in the multiple scattering expansion of the Faddeev amplitude, while the short-dashed curve (R 2) includes the first two terms, and the dash-dotted curve (R 3) the first three terms. The data are taken from Ref. [17. 

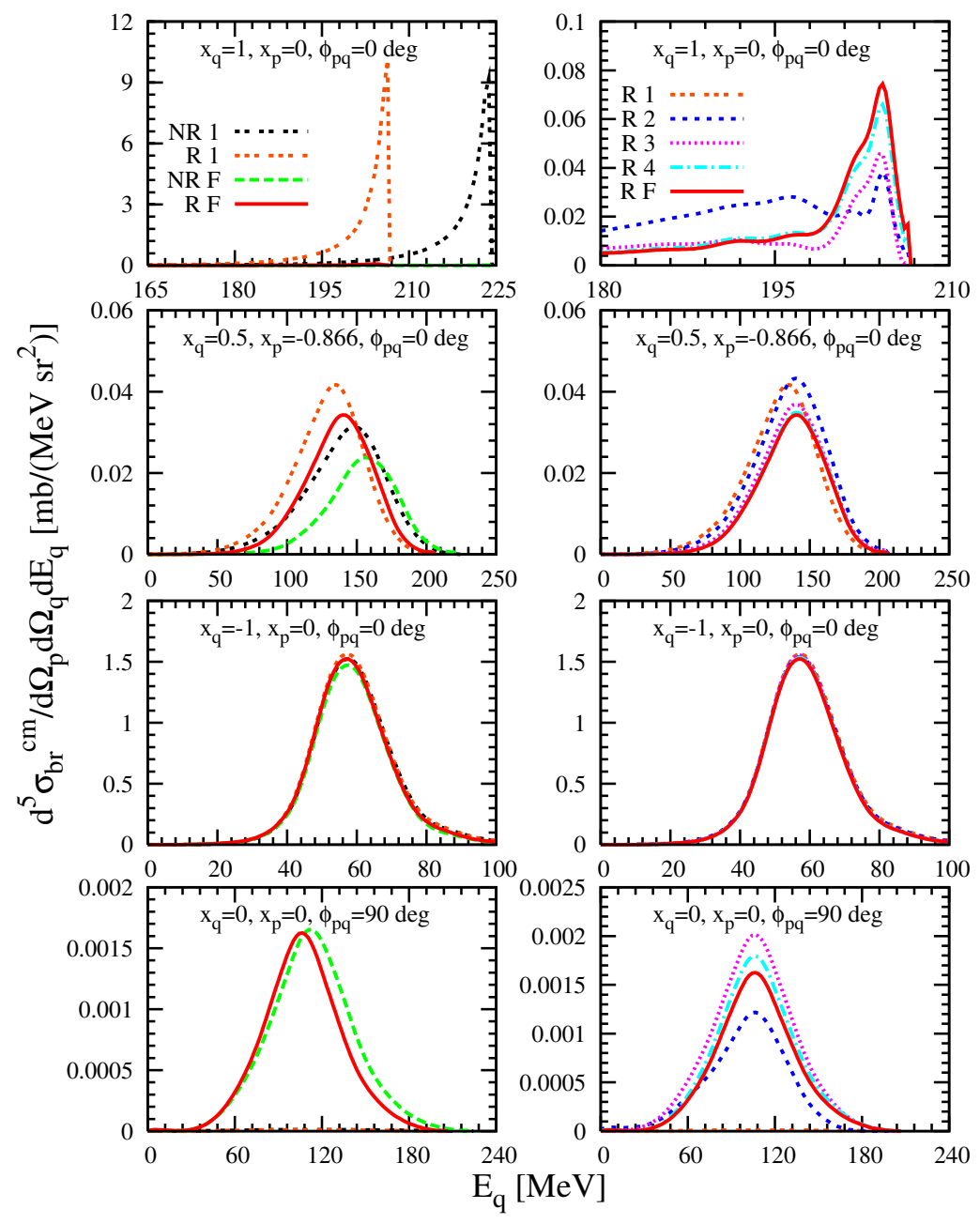

Fig. 4. The exclusive differential cross section for the ${ }^{2} \mathrm{H}(\mathrm{p}, 2 \mathrm{p}) \mathrm{n}$ reaction at $508 \mathrm{MeV}$ laboratory projectile energy for different star configurations as function of the c.m. kinetic energy of one of the outgoing protons for different c.m. angle pairs $x_{q}=\cos \theta_{q}, x_{p}=\cos \theta_{p}$. The angle $\phi_{p q}=0$ deg indicates coplanar star configurations, while $\phi_{p q}=90 \mathrm{deg}$ indicates a space star. All figures show the full relativistic Faddeev calculation as solid line ( R F). The left column compares the full relativistic calculation with the full nonrelativistic one (NR F), as well as the corresponding relativistic (R 1) and nonrelativistic (NR 1) first order calculations. The right column shows the subsequent sum of the lowest orders in the multiple scattering series contributing to the full relativistic result. The meaning of those curves is the same as in Fig. 3 